\title{
Focal adhesion kinase is overexpressed in thymic epithelial tumors and may serve as an independent prognostic biomarker
}

\author{
MENG LI ${ }^{1}$, FENG HOU ${ }^{2}$, JIE ZHAO ${ }^{2}$, TIANQIANG ZHANG ${ }^{3}$, \\ DONGFEI LI ${ }^{1}$, WENSI WU ${ }^{1}$, XIAOTONG LIU ${ }^{1}$ and LINHAO XU ${ }^{1}$
}

Departments of ${ }^{1}$ Thoracic Surgery and ${ }^{2}$ Pathology, The Affiliated Hospital of Qingdao University, Qingdao, Shandong 266003;

${ }^{3}$ Department of Cardiothoracic Surgery, The Municipal Hospital of Zaozhuang, Zaozhuang, Shandong 277100, P.R. China

Received December 6, 2016; Accepted November 2, 2017

DOI: $10.3892 / \mathrm{ol} .2017 .7676$

\begin{abstract}
Focal adhesion kinase (FAK) has long been considered to be a key regulator of growth factor receptor- and integrin-mediated signals, with pivotal roles in tumor cells through its kinase activity and scaffolding function. Increased FAK expression and activity has been observed in tumors of various origins and is often associated with a poor prognosis. However, there have been no studies on the aberrant expression of FAK in thymic epithelial tumors to date. The aim of the present study was to evaluate FAK expression in thymic epithelial tumors and to explore the prognostic significance of FAK. FAK expression was investigated in 100 formalin-fixed, paraffin-embedded human thymic epithelial tumor (TET) specimens using immunohistochemical analysis with FAK-specific monoclonal antibody 4.47, and the associations between FAK expression and clinicopathological parameters (including sex, age, tumor size, myasthenia gravis, World Health Organization classification and Masaoka-Koga stage) were analyzed. FAK was significantly overexpressed in TETs compared with in normal thymus tissues $(\mathrm{P}<0.001)$. Additionally, FAK overexpression was significantly associated with advanced tumor stages (stages III or IV; $\mathrm{P}<0.001$ ) and highly aggressive TET subtypes (type B2 and B3 thymomas and thymic carcinomas; $\mathrm{P}<0.001)$. Furthermore, FAK overexpression was significantly associated with a worse 10 -year overall survival, as determined by univariate analysis $(\mathrm{P}<0.001)$. Multivariate analysis revealed that FAK overexpression was an independent prognostic factor for patients with TETs $(\mathrm{P}=0.034)$. The results of the present study suggest that FAK serves an important role in the tumorigenesis and progression of TETs. Therefore, FAK may serve as a prognostic biomarker and is a potential therapeutic target for the treatment of TETs.
\end{abstract}

Correspondence to: Professor Linhao Xu, Department of Thoracic Surgery, The Affiliated Hospital of Qingdao University, 16 Jiangsu Road, Qingdao, Shandong 266003, P.R. China

E-mail: qdqyfyxulinhao@126.com

Key words: focal adhesion kinase, thymic epithelial tumor, immunohistochemistry, prognosis, mediastinal tumor

\section{Introduction}

Thymic epithelial tumors (TETs), well known for their variability in morphological appearance and for the heterogeneity of their neoplastic epithelial cells, are epithelial neoplasms originating from the thymus $(1,2)$. TETs account for $50 \%$ of the anterior mediastinal masses occurring in the adult population and represent the most common tumors of the anterior portion of the mediastinum (3). However, the overall incidence of TETs is rare, at 3.2 cases/1,000,000 individuals per year (4). The majority of patients are between the ages of 40 and 60 years at the time of diagnosis, with no significant difference between males and females with respect to the incidence of this disease (5). Furthermore, previous studies have demonstrated that TETs are associated with a variety of autoimmune disorders, including myasthenia gravis, pure red cell aplasia and hypogammaglobulinemia $(3,5)$.

According to the widely-accepted World Health Organization (WHO) classification system, which is based upon the morphological features and atypia of epithelial cells as well as the lymphocyte-to-epithelial cell ratio, TETs are subdivided into thymomas (types A, AB, B1, B2 and B3) and thymic carcinomas (TCs) (6). Thymomas are generally considered to have an indolent growth pattern with malignant transformation potential. Certain thymomas demonstrate local invasion, pleural dissemination and systemic metastasis during advanced stages, despite being indolent and non-invasive during their early stages (1). Furthermore, previous studies have documented the recurrence and metastasis of thymomas following resection (7-9), suggesting that even unaggressive, non-invasive thymomas possess the fundamental features of a malignant tumor. Therefore, thymomas require consideration as a potentially malignant disease requiring prolonged follow-up, despite the fact that the majority are low grade and indolent (10). TCs, as the most aggressive subtype of TETs, usually exhibit a more invasive phenotype with a worse survival rate, as well as a greater potential for relapse and metastasis compared with the majority of thymomas (11). Surgical resection remains the optimal treatment for early-stage TETs, while advanced-stage, unresectable or recurrent thymic malignancies are usually treated with palliative chemotherapy (12).

However, due to the rarity and morphological complexity of TETs, the biological mechanisms that facilitate TET 
tumorigenesis and development remain unclear at present. Additionally, biomarkers that are associated with clinical behavior and prognosis are urgently required.

Tumor invasion and metastasis are complex processes involving interactions between cancer cells and the extracellular matrix (ECM), including alterations in cell adhesion and motility that permit tumor cells to invade and migrate through the ECM (13). A number of these alterations develop at the contact points between cells and the ECM, which are known as focal adhesions. Focal adhesion kinase (FAK), originally identified in v-Src-transformed chicken embryo fibroblasts (14), is a highly conserved $125-\mathrm{kDa}$ cytoplasmic, non-receptor protein tyrosine kinase that is activated and localized at the sites of cellular focal adhesions (15). As a critical mediator of signaling events between cells and the ECM, FAK serves a pivotal function in growth factor receptor- and integrin-mediated signal transduction pathways (16). The activation of FAK by integrin clustering, cell adhesion or growth factor receptors induces the rapid phosphorylation of FAK at Tyr-397, which has been identified as the major autophosphorylation site. Once phosphorylated, Tyr-397 generates a high-affinity binding site for SH2-domain-containing proteins, including Src-family kinases, phospholipase $\mathrm{Cg}$ and growth factor receptor-bound protein 7 (17). The subsequent binding of FAK to Src likely contributes to Src kinase activation, which in turn promotes the phosphorylation of FAK at additional tyrosine residues to stimulate maximal FAK kinase activity (18).

FAK has been implicated in the regulation of a diverse set of cellular functions, including survival, migration, proliferation, angiogenesis and apoptosis, in a variety of cell types (16), suggesting that FAK may contribute to tumor formation and malignant progression (19). Previously, FAK has also been demonstrated to serve an important role in the regulation of cancer stem cells, the epithelial-to-mesenchymal transition and the tumor microenvironment $(16,20)$. In addition, accumulating evidence has demonstrated that FAK is overexpressed in a wide range of human tumors, including colon, breast, oral, liver, head and neck tumors, gastric carcinomas and neuroblastomas (21-28). Furthermore, the overexpression of FAK has been reported to serve as an independent prognostic factor for various types of malignancies, including ovarian, esophageal and hepatocellular cancer, as well as acute myeloid leukemia (29-32).

However, despite the gradually-increasing research into various malignancies, there is insufficient data available regarding FAK expression in TETs. Therefore, the present study aimed to examine FAK expression in TETs and to determine whether FAK expression is associated with the clinical behavior and prognosis of TETs.

\section{Materials and methods}

Patients and specimens. TET tissue specimens were obtained from 100 patients who had undergone tumor resection without preoperative chemotherapy or radiotherapy at the Department of Thoracic Surgery, Affiliated Hospital of Qingdao University (Qingdao, China) between January 2002 and December 2006. Of the 100 patients who were included in the study, 55 were male and 45 were female (male-to-female ratio of 1.22:1) and the mean age of the patients was $52.1 \pm 13.7$ years (range,
19-80 years). Additionally, 28 patients (28.0\%) suffered from myasthenia gravis. Tumor diameters ranged between 1.5 and $11.0 \mathrm{~cm}$, with a mean diameter of $5.8 \pm 2.2 \mathrm{~cm}$. TETs were classified into 84 thymomas ( 9 type A, 24 type AB, 15 type B1, 18 type $\mathrm{B} 2$ and 18 type B3) and $16 \mathrm{TCs}$, according to the 2015 WHO criteria (6). Furthermore, clinical stages were based upon the newly revised Masaoka-Koga staging system (10), with 37 stage I, 27 stage II, 23 stage III and 13 stage IV cases in the study cohort. In addition, 58 normal thymus tissues were obtained from different patients with mediastinal cysts who had undergone mediastinal cystectomy at the same institution between January 2002 and December 2006. The normal thymus tissues were used as the control group. The healthy thymus cohort consisted of 28 men and 30 women, aged 18-72 years (mean, 48.6 \pm 13.2 years). The medical records and histopathological archives of all patients were complete. Diagnoses were confirmed histologically in all specimens, based upon the examination of sections stained with hematoxylin and eosin at the Department of Pathology of the Affiliated Hospital of Qingdao University. Following surgical resection, all specimens were fixed in $10 \%$ buffered formalin overnight at room temperature prior to being embedded in paraffin. Postoperative follow-up data were obtained from all patients, with a mean follow-up period of 101.3 \pm 31.1 months (range, 3-120 months). One patient with type AB thymoma did not complete the follow-up. The present study was performed in accordance with the Declaration of Helsinki, and was approved by the Medical Ethics Committees of the Affiliated Hospital of Qingdao University. Written informed consent was obtained from all participants included in the study.

Immunohistochemistry. Formalin-fixed, paraffin-embedded tissue sections were cut into $4-\mu \mathrm{m}$ thick sections. Following routine deparaffinization and rehydration in descending ethanol series $(100,95,85$ and $70 \%)$ for $5 \mathrm{~min}$, respectively, the sections were autoclaved in citrate buffer ( $\mathrm{pH}$ 6.0; $0.1 \mathrm{M} ; 1$ 1; Zhongshan Goldenbridge Biotechnology Co., Ltd., Beijing, China) for $5 \mathrm{~min}$ at $120^{\circ} \mathrm{C}$ for antigen retrieval. Sections were then washed in PBS three times. Endogenous peroxidase activity was blocked in methanol containing $0.3 \% \mathrm{H}_{2} \mathrm{O}_{2}$ for $30 \mathrm{~min}$ at room temperature. The sections were subsequently washed in PBS (3 times, for 3 min each time), prior to being incubated with a mouse anti-FAK monoclonal primary antibody 4.47 (dilution 1:200; cat. no. 05-537; EMD Millipore, Billerica, MA, USA) overnight at $4^{\circ} \mathrm{C}$. Following 3 cycles of washing with PBS (for 3 min each time), the sections were incubated with the Polink-1 horseradish peroxidase mouse for DAB Bulk kit (cat. no. D12-110; Golden Bridge International, Inc., Bothell, WA, USA), at room temperature for $15 \mathrm{~min}$. All procedures were performed according to the manufacturer's instructions. Sections were washed again with PBS (3 times for 3 min each time), prior to being treated at room temperature with a diaminobenzidine working solution for $10 \mathrm{~min}$ and then counterstained with hematoxylin at room temperature for $5 \mathrm{~min}$. The negative controls underwent the same procedures; however, the primary antibody was omitted or replaced with normal serum.

Evaluation of immunohistochemistry. The presence of staining was evaluated by two board-certified pathologists who were blinded to the clinical data of the patients. FAK 

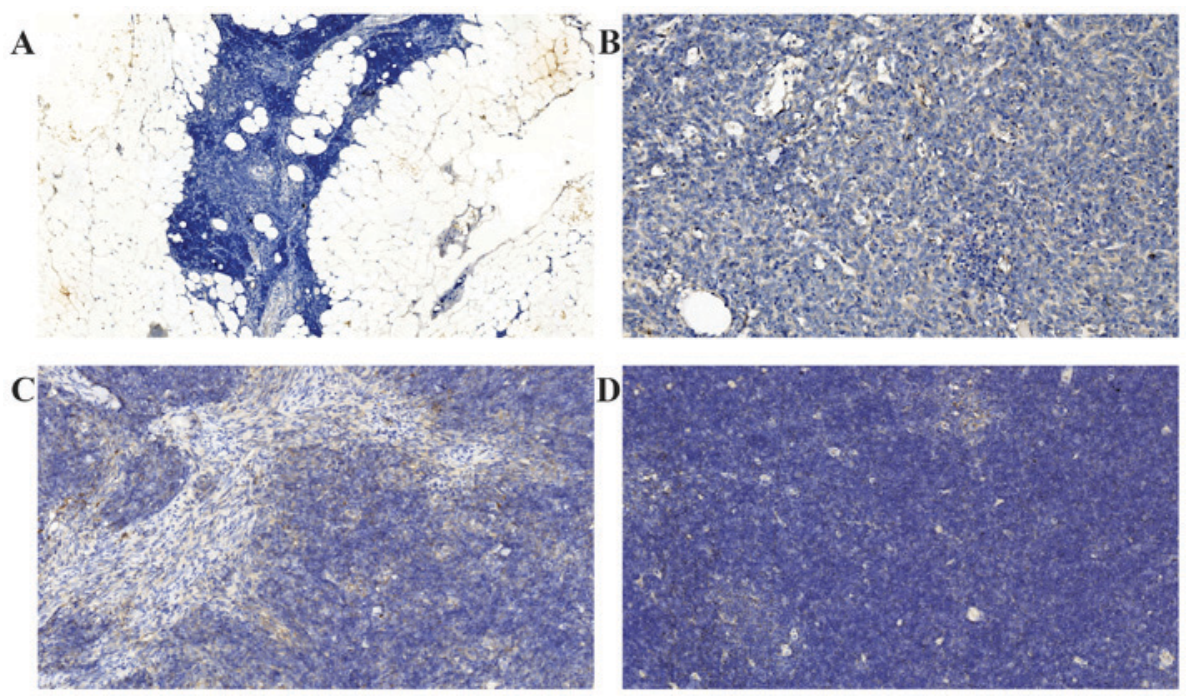

Figure 1. Representative photomicrographs of FAK immunostaining in normal thymus and less aggressive subtypes of thymic epithelial tumor. (A) Normal thymus tissues were negative for FAK expression (magnification, x100). Weak expression of FAK was observed in (B) type A, (C) type AB and (D) type B1 thymomas (magnification, $\mathrm{x} 200$ ). FAK, focal adhesion kinase.
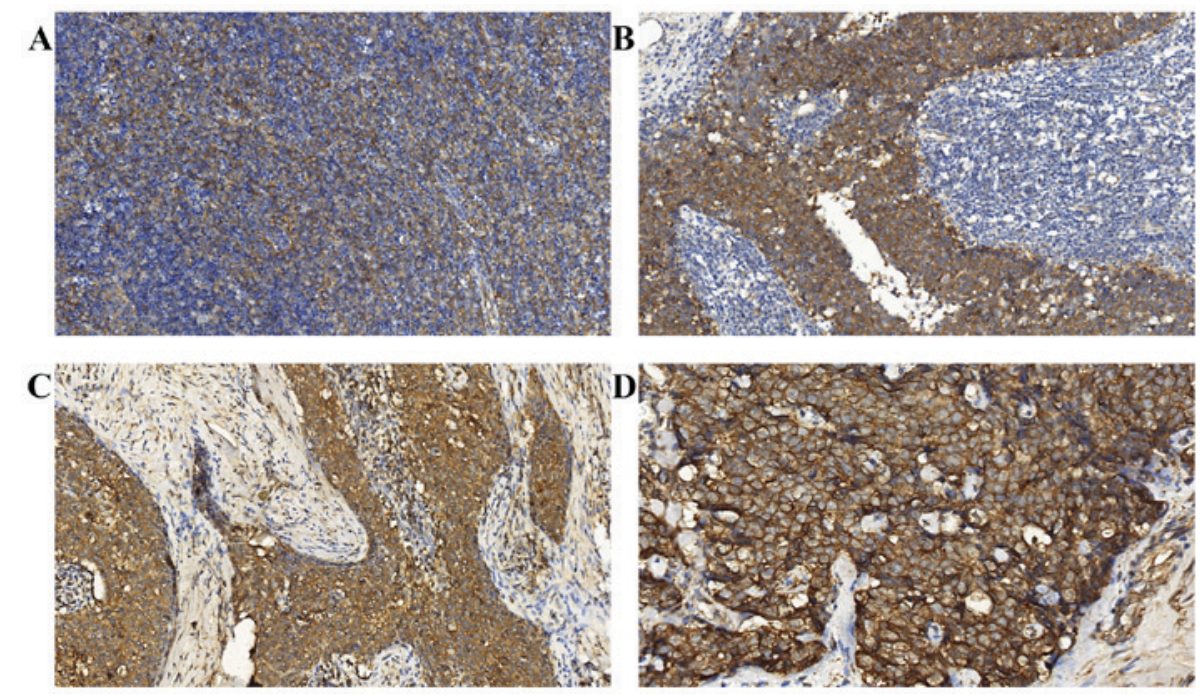

Figure 2. Representative photomicrographs of FAK immunostaining in highly aggressive subtypes of thymic epithelial tumor. FAK was overexpressed in (A) type B2 and (B) type B3 thymomas, and in (C) thymic carcinoma (magnification, x200). (D) A high-power view of FAK overexpression in thymic carcinoma epithelial cells, demonstrating the intense membrane and cytoplasmic immunoreactivity for FAK (magnification, $\mathrm{x} 400$ ). FAK, focal adhesion kinase.

expression was determined using a scoring system that measured staining intensity ( 0 , negative; 1 , weak; 2 , moderate; and 3 , intense) and the proportion of positively-stained cells among the tumor epithelial cells ( 0 , none; $1,1-25 \% ; 2,26-50 \%$; $3,51-75 \%$; and $4,76-100 \%)$ in $\geq 5$ areas under a light microscope (Olympus BX41; Olympus Corporation, Tokyo, Japan) at X400 magnification. The overall staining score was obtained by multiplying the staining intensity score by the score representing the proportion of positive-stained cells. Specimens were separated into high expression (overall score $\geq 6$ ) and low expression (overall score $<6$ ) groups to more accurately determine FAK expression.

Statistical analysis. The $\chi^{2}$ test and the Kruskal-Wallis test were used to assess the association between FAK expression and different clinicopathological parameters. Overall survival rates were calculated using the Kaplan-Meier method, and the differences between the survival curves were evaluated using the log-rank test. A Cox proportional hazards multivariate regression model was developed to identify independent significant prognostic factors. $\mathrm{P}<0.05$ was considered to indicate a statistically significant difference. SPSS 22.0 software (IBM Corp., Armonk, NY, USA) was used to perform all analyses.

\section{Results}

FAK is expressed in TETs but not in the normal thymus. All normal thymus tissues were negative for FAK expression (Fig. 1A). Positive immunostaining of FAK was detected at varying levels in all the TET specimens. FAK staining was localized to the cytoplasm and the membrane of the epithelial cells of the thymic tumors (Figs. 1B-D and 2A-D). Notably, the 
Table I. Association between FAK expression and clinicopathological parameters in 100 patients with thymic epithelial tumors.

\begin{tabular}{|c|c|c|c|c|}
\hline \multirow[b]{2}{*}{ Parameter } & \multirow[b]{2}{*}{ Total, $\mathrm{n}$} & \multicolumn{2}{|c|}{ FAK expression } & \multirow[b]{2}{*}{$\mathrm{P}$-value } \\
\hline & & High $(n=52)$ & Low $(n=48)$ & \\
\hline Age, years & & & & 0.986 \\
\hline$\leq 60$ & 73 & 38 & 35 & \\
\hline$>60$ & 27 & 14 & 13 & \\
\hline Sex & & & & 0.520 \\
\hline Male & 55 & 27 & 28 & \\
\hline Female & 45 & 25 & 20 & \\
\hline Tumor size & & & & 0.097 \\
\hline$\leq 6 \mathrm{~cm}$ & 56 & 25 & 31 & \\
\hline$>6 \mathrm{~cm}$ & 44 & 27 & 17 & \\
\hline MG & & & & 0.844 \\
\hline Present & 28 & 15 & 13 & \\
\hline Absent & 72 & 37 & 35 & \\
\hline Classification & & & & $<0.001$ \\
\hline $\mathrm{A}, \mathrm{AB}, \mathrm{B} 1$ & 48 & 10 & 38 & \\
\hline $\mathrm{B} 2, \mathrm{~B} 3, \mathrm{TC}$ & 52 & 42 & 10 & \\
\hline Stage & & & & $<0.001^{\mathrm{a}}$ \\
\hline I & 37 & 7 & 30 & \\
\hline II & 27 & 12 & 15 & \\
\hline III & 23 & 20 & 3 & \\
\hline IV & 13 & 13 & 0 & \\
\hline
\end{tabular}

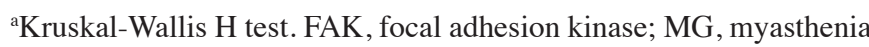
gravis; TC, thymic carcinoma.

tumor lymphocytes did not express FAK. Significantly higher FAK expression was observed in $52.0 \%$ of TETs $(52 / 100)$ compared with in the normal thymus, as determined using the $\chi^{2}$ test $(\mathrm{P}<0.001)$. Furthermore, FAK was weakly expressed in the majority of the less aggressive TET subtypes (types A, $\mathrm{AB}$ and $\mathrm{B} 1$ thymomas), but was upregulated in the majority of highly aggressive tumor subtypes (types B2 and B3 thymomas, and TCs).

Associations between FAK overexpression and clinicopathological parameters in TETs. The associations between the clinicopathological characteristics of patients with TETs and FAK overexpression are summarized in Table I. A statistically significant association was observed between FAK overexpression and the Masaoka-Koga stage and WHO classification (both $\mathrm{P}<0.001)$. Notably, 33/36 $(91.7 \%)$ high-stage (stages III and IV) tumors overexpressed FAK, compared with only 19/64 (29.7\%) low-stage (stages I and II) tumors $(\mathrm{P}<0.001)$. In the less aggressive TET subtypes, the majority of tumors exhibited low FAK expression, with only 10/48 tumors (20.8\%) exhibiting high FAK expression. By contrast, in the highly aggressive subtypes of TETs, 42/52 tumors (80.8\%) exhibited high FAK expression. This difference between the two groups was statistically significant $(\mathrm{P}<0.001)$. However,
Table II. Univariate analyses of prognostic factors in patients with thymic epithelial tumors.

\begin{tabular}{|c|c|c|c|}
\hline Parameter & Total, $\mathrm{n}$ & 10-year OS, \% & P-value \\
\hline Age, years & & & 0.100 \\
\hline$\leq 60$ & 73 & 71.2 & \\
\hline$>60$ & 27 & 54.9 & \\
\hline Sex & & & 0.645 \\
\hline Male & 55 & 65.5 & \\
\hline Female & 45 & 68.6 & \\
\hline Tumor size & & & 0.023 \\
\hline$\leq 6 \mathrm{~cm}$ & 56 & 76.7 & \\
\hline$>6 \mathrm{~cm}$ & 44 & 54.5 & \\
\hline FAK expression & & & $<0.001$ \\
\hline Low & 48 & 93.7 & \\
\hline High & 52 & 42.3 & \\
\hline MG & & & 0.278 \\
\hline Absent & 72 & 63.7 & \\
\hline Present & 28 & 75.0 & \\
\hline Classification & & & $<0.001$ \\
\hline $\mathrm{A}, \mathrm{AB}, \mathrm{B} 1$ & 48 & 91.5 & \\
\hline B2, B3, TC & 52 & 44.2 & \\
\hline Stage & & & $<0.001$ \\
\hline I and II & 64 & 89.0 & \\
\hline III and IV & 36 & 27.8 & \\
\hline
\end{tabular}

OS, overall survival; FAK, focal adhesion kinase; MG, myasthenia gravis; TC, thymic carcinoma.

FAK expression was not associated with age, sex, tumor size or myasthenia gravis (Table I).

Univariate and multivariate analyses of prognostic factors for patients with TETs. The 10-year overall survival rates of patients were analyzed in order to assess the prognostic significance of FAK expression, as well as that of other clinicopathological parameters (Table II). The 10-year survival rate of the high FAK expression group was significantly lower than that for the low FAK expression group in patients with TETs, as determined using the log rank test (42.3 vs. 93.7\%; $\mathrm{P}<0.001$; Fig. 3). Multivariate analysis, using the Cox proportional hazards model, of the WHO classification, Masaoka-Koga stage, tumor size and FAK expression revealed that only high FAK expression and the Masaoka-Koga stage were significant independent prognostic factors of TETs $(\mathrm{P}=0.034$ and $\mathrm{P}=0.005$, respectively; Table III).

\section{Discussion}

FAK, an important mediator between cells and the extracellular matrix, is considered to serve an important function in a number of biological processes, including cell adhesion, spreading, motility, proliferation, survival, apoptosis and migration (33). Given that the dysregulation of these processes is often associated with the development and progression 
Table III. Multivariate analyses of prognostic factors in patients with thymic epithelial tumors.

\begin{tabular}{lccc}
\hline Parameter & P-value & HR & 95\% CI \\
\hline FAK expression (low/high) & 0.034 & 4.080 & $1.110-15.005$ \\
Stage (I+II/III+IV) & 0.005 & 3.824 & $1.506-9.713$ \\
Classification (A+AB+B1/B2+B3+TC) & 0.110 & 2.548 & $0.808-8.032$ \\
Tumor size $(\leq 6 />6 \mathrm{~cm})$ & 0.161 & 1.653 & $0.818-3.338$ \\
\hline
\end{tabular}

HR, hazard ratio; CI, confidence interval; FAK, focal adhesion kinase; TC, thymic carcinoma.

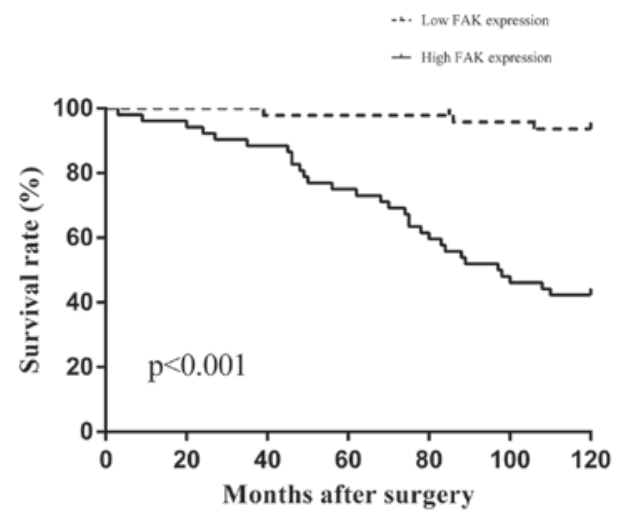

Figure 3. The 10-year overall survival curves of patients with thymic epithelial tumors. High expression of FAK was significantly associated with a worse prognosis $(\mathrm{P}<0.001, \log$-rank test). FAK, focal adhesion kinase.

of tumors, it is understandable that FAK is highly involved in human malignancies. Increased expression and elevated activity of FAK have been demonstrated by previous studies to be associated with clinicopathological features in a wide range of human cancer types $(21-23,27,30,32,34)$, thereby suggesting a role for FAK in carcinogenesis. However, to the best of our knowledge, there has been no previous study on FAK expression in TETs, which are rare and histologically complex tumors. Therefore, the present study analyzed FAK expression and assessed its clinical value as a prognostic marker in TETs.

The results of the present study provided definitive evidence that FAK is not expressed in the normal thymus but is significantly upregulated in TETs, as determined by immunohistochemical analysis, indicating that FAK overexpression is associated with the tumorigenesis of TETs. The present study also evaluated the association between FAK expression and clinicopathological parameters in TETs. Notably, the results of the present study demonstrated that FAK overexpression was not only strongly associated with advanced stages of TETs, but was also associated with highly aggressive subtypes. Therefore, it was hypothesized that FAK may serve important functions in the invasion and metastasis of TETs. However, no statistical association was observed between FAK expression and tumor size, sex, age or myasthenia gravis in the present study.

The observation that elevated levels of FAK are detected in TETs is consistent with reports from previous studies of FAK overexpression in other types of solid tumor. For example, Cance et al (21) analyzed the expression of FAK in normal, pre-invasive and invasive human breast and colon tissues from individual patients using immunohistochemistry. In this study, FAK was weakly expressed in the majority of benign breast and colon epithelia but was overexpressed in the majority of invasive breast carcinomas and colon cancer tissues, suggesting that FAK overexpression may occur in the early stages of tumorigenesis (21). Additionally, Lark et al (23) observed elevated levels of FAK expression in primary colorectal carcinomas and liver metastases compared with matched healthy colorectal tissues, using quantitative polymerase chain reaction (qPCR) and immunohistochemical analyses. The results of the aforementioned study suggested that the increased expression of FAK may suppress apoptosis and promote the survival and metastasis of cancer cells (23). Furthermore, Itoh et al (22) used western blotting and immunohistochemical analyses to demonstrate that FAK expression was upregulated in hepatocellular carcinoma (HCC), and that it was significantly associated with portal venous invasion, indicating that FAK overexpression serves an important function in HCC progression and invasion (22). Another study used immunohistochemistry and reverse transcription qPCR to demonstrate that FAK was overexpressed in non-small cell lung cancer (NSCLC) and that its overexpression was associated with nodal metastasis and advanced stages of NSCLC, suggesting that FAK serves an important role in lung cancer progression and metastasis (35).

Notably, the present study also used univariate and multivariate analyses to demonstrate that the overexpression of FAK in patients with TETs is significantly associated with a worse overall survival rate. Therefore, these results indicated that FAK overexpression may be an independent prognostic biomarker for TETs. The results of the present study are consistent with those of previous studies that have demonstrated FAK protein overexpression to be an independent unfavorable prognostic factor in various types of tumor (22,29-32). By contrast, it has also been reported that FAK expression was not associated with prognosis in certain types of tumor $(25,26,34)$. Taken together, the results of these studies suggest that FAK may serve distinct roles in different types of malignancies and during different stages of tumor progression.

In the present study, the marked upregulation of FAK in TETs, particularly in the advanced stages and highly aggressive tumor subtypes, combined with the negative expression of FAK in normal thymus tissues, suggests that FAK may be a potential therapeutic target for TETs. Recently, a FAK-targeting therapeutic approach has focused primarily on blocking its kinase enzymatic activity and targeting its scaffolding function using pharmacological agents; a number of FAK-directed 
small molecule inhibitors, including PF-00562271, VS-4718, GSK2256098, BI853520 and PF-04554878, are undergoing clinical trials in patients with cancer (16). Furthermore, a combination therapy approach using FAK inhibitors against FAK signaling pathways (e.g., the phosphatidylinositol-3-kinase, protein kinase B, epidermal growth factor receptor, human epidermal growth factor receptor-2, Src, mitogen-activated protein kinase and cellular-mesenchymal-to-epithelial transition factor signaling pathways) was employed to sensitize cancer cells to chemotherapy and to increase the efficacy of drugs $(16,33)$. Considering this, the therapeutic utility of FAK inhibitors in the advanced stages of TETs remains promising. However, future clinical trials are required in order to evaluate the clinical efficacy of these approaches in the treatment of TETs.

In the present study, immunohistochemistry was performed using a monoclonal FAK-specific 4.47 antibody, which was revealed to be highly specific to human FAK in formalin-fixed, paraffin-embedded tissue sections (21). This antibody is able to recognize the FAK-specific epitope at the amino terminus of a molecule in order to avoid consensus sequences within the kinase domain and cross-reactivity with the FAK-related non-kinase, an autonomously expressed carboxyl-terminal fragment. Furthermore, patients who had received adjuvant preoperative chemotherapy or radiotherapy were excluded from the present study in order to eliminate the potential effects of these treatments on the immunohistochemistry results. However, the sample sizes were relatively small in the present study due to the low incidence of TETs. Therefore, further studies comprising larger cohorts in multiple treatment centers are required in order to confirm these results.

In conclusion, to the best of our knowledge, the present study is the first to provide definitive evidence that FAK expression is upregulated in TETs. FAK overexpression may serve an important role in the tumorigenesis and progression of TETs. Furthermore, the results of the present study indicated that FAK overexpression may be used as a prognostic biomarker for TET. Additionally, FAK may be a promising therapeutic target for TETs, but the precise role of FAK in TETs remains to be confirmed by further in vivo and in vitro studies.

\section{References}

1. Masaoka A, Monden Y, Nakahara K and Tanioka T: Follow-up study of thymomas with special reference to their clinical stages. Cancer 48: 2485-2492, 1981.

2. Chen G, Marx A, Chen WH, Yong J, Puppe B, Stroebel P and Mueller-Hermelink HK: New WHO histologic classification predicts prognosis of thymic epithelial tumors: A clinicopathologic study of 200 thymoma cases from China. Cancer 95: 420-429, 2002

3. Venuta F, Anile M, Diso D, Vitolo D, Rendina EA, De Giacomo T, Francioni $\mathrm{F}$ and Coloni GF: Thymoma and thymic carcinoma. Eur J Cardiothoracic Surg 37: 13-25, 2010.

4. de Jong WK, Blaauwgeers JL, Schaapveld M, Timens W, Klinkenberg TJ and Groen HJ: Thymic epithelial tumours: A population-based study of the incidence, diagnostic procedures and therapy. Eur J Cancer 44: 123-130, 2008.

5. Wright CD: Management of thymomas. Crit Rev Oncol Hematol 65: 109-120, 2008.

6. Marx A, Chan JK, Coindre JM, Detterbeck F, Girard N, Harris NL, Jaffe ES, Kurrer MO, Marom EM, Moreira AL, et al The 2015 World Health Organization Classification of tumors of the thymus: Continuity and changes. J Thorac Oncol 10: $1383-1395,2015$.
7. Gamboa EO, Sawhney V, Lanoy RS, Haller NA, Powell AT and Hazra SV: Widespread metastases after resection of noninvasive thymoma. J Clin Oncol 26: 1752-1755, 2008.

8. Jain RK, Mehta RJ, Henley JD, Kesler KA, Loehrer PJ and Badve S: WHO types A and AB thymomas: Not always benign. Mod Pathol 23: 1641-1649, 2010.

9. Okumura M, Shiono H, Inoue M, Tanaka H, Yoon HE, Nakagawa K, Matsumura A, Ohta M, Iuchi K and Matsuda H: Outcome of surgical treatment for recurrent thymic epithelial tumors with reference to world health organization histologic classification system. J Surg Oncol 95: 40-44, 2007.

10. Detterbeck FC, Nicholson AG, Kondo K, Van Schil PV and Moran C: The Masaoka-Koga stage classification for thymic malignancies: Clarification and definition of Terms. J Thorac Oncol 6 (7 Suppl 3): S1710-S1716, 2011

11. Ogawa K, Toita T, Uno T, Fuwa N, Kakinohana Y, Kamata M, Koja K, Kinjo T, Adachi G and Murayama S: Treatment and prognosis of thymic carcinoma: A retrospective analysis of 40 cases. Cancer 94: 3115-3119, 2002.

12. Thomas A, Rajan A, Berman A, Tomita Y, Brzezniak C, Lee MJ, Lee S, Ling A, Spittler AJ, Carter CA, et al: Sunitinib in patients with chemotherapy-refractory thymoma and thymic carcinoma: An open-label phase 2 trial. Lancet Oncol 16: 177-186, 2015.

13. Liotta LA and Stetler-Stevenson WG: Tumor invasion and metastasis: An imbalance of positive and negative regulation. Cancer Res 51 (Suppl 18): 5054S-5059S, 1991.

14. Schaller MD, Borgman CA, Cobb BS, Vines RR, Reynolds AB and Parsons JT: pp125FAK a structurally distinctive protein-tyrosine kinase associated with focal adhesions. Proc Natl Acad Sci USA 89: 5192-5196, 1992.

15. Hanks SK, Calalb MB, Harper MC and Patel SK: Focal adhesion protein-tyrosine kinase phosphorylated in response to cell attachment to fibronectin. Proc Natl Acad Sci USA 89: 8487-8491, 1992.

16. Lee BY, Timpson P, Horvath LG and Daly RJ: FAK signaling in human cancer as a target for therapeutics. Pharmacol Ther 146: 132-149, 2015.

17. Mitra SK, Hanson DA and Schlaepfer DD: Focal adhesion kinase: In command and control of cell motility. Nat Rev Mol Cell Biol 6: 56-68, 2005.

18. Dwyer SF, Gao L and Gelman IH: Identification of novel focal adhesion kinase substrates: Role for FAK in $\mathrm{NF} \kappa \mathrm{B}$ signaling. Int J Biol Sci 11: 404-410, 2015.

19. McLean GW, Carragher NO, Avizienyte E, Evans J, Brunton VG and Frame MC: The role of focal-adhesion kinase in cancer - a new therapeutic opportunity. Nat Rev Cancer 5: 505-515, 2005.

20. Sulzmaier FJ, Jean C and Schlaepfer DD: FAK in cancer: Mechanistic findings and clinical applications. Nat Rev Cancer 14: 598-610, 2014.

21. Cance WG, Harris JE, Iacocca MV, Roche E, Yang X, Chang J, Simkins $\mathrm{S}$ and $\mathrm{Xu} \mathrm{L}$ : Immunohistochemical analyses of focal adhesion kinase expression in benign and malignant human breast and colon tissues: Correlation with preinvasive and invasive phenotypes. Clin Cancer Res 6: 2417-2423, 2000.

22. Itoh S, Maeda T, Shimada M, Aishima S, Shirabe K, Tanaka S and Maehara Y: Role of expression of focal adhesion kinase in progression of hepatocellular carcinoma. Clin Cancer Res 10: 2812-2817, 2004

23. Lark AL, Livasy CA, Calvo B, Caskey L, Moore DT, Yang X and Cance WG: Overexpression of focal adhesion kinase in primary colorectal carcinomas and colorectal liver metastases: Immunohistochemistry and real-time PCR analyses. Clin Cancer Res 9: 215-222, 2003.

24. Owens LV, Xu L, Craven RJ, Dent GA, Weiner TM, Kornberg L, Liu ET and Cance WG: Overexpression of the focal adhesion kinase (p125FAK) in invasive human tumors. Cancer Res 55: 2752-2755, 1995.

25. Beierle EA, Massoll NA, Hartwich J, Kurenova EV, Golubovskaya VM, Cance WG, McGrady P and London WB: Focal adhesion kinase expression in human neuroblastoma: Immunohistochemical and real-time PCR analyses. Clin Cancer Res 14: 3299-3305, 2008.

26. Canel M, Secades P, Rodrigo JP, Cabanillas R, Herrero A, Suarez C and Chiara MD: Overexpression of focal adhesion kinase in head and neck squamous cell carcinoma is independent of fak gene copy number. Clin Cancer Res 12: 3272-3279, 2006.

27. Schneider GB, Kurago Z, Zaharias R, Gruman LM, Schaller MD and Hendrix MJ: Elevated focal adhesion kinase expression facilitates oral tumor cell invasion. Cancer 95: 2508-2515, 2002. 
28. Lai IR, Chu PY, Lin HS, Liou JY, Jan YJ, Lee JC and Shen TL: Phosphorylation of focal adhesion kinase at Tyr397 in gastric carcinomas and its clinical significance. Am J Pathol 177: $1629-1637,2010$

29. Recher C, Ysebaert L, Beyne-Rauzy O, Mansat-De Mas V Ruidavets JB, Cariven P, Demur C, Payrastre B, Laurent G and Racaud-Sultan C: Expression of focal adhesion kinase in acute myeloid leukemia is associated with enhanced blast migration, increased cellularity, and poor prognosis. Cancer Res 64: 3191-3197, 2004

30. Sood AK, Coffin JE, Schneider GB, Fletcher MS, DeYoung BR, Gruman LM, Gershenson DM, Schaller MD and Hendrix MJ: Biological significance of focal adhesion kinase in ovarian cancer: Role in migration and invasion. Am J Pathol 165: 1087-1095, 2004.

31. Fujii T, Koshikawa K, Nomoto S, Okochi O, Kaneko T, Inoue S, Yatabe Y, Takeda S and Nakao A: Focal adhesion kinase is overexpressed in hepatocellular carcinoma and can be served as an independent prognostic factor. J Hepatol 41: 104-111, 2004.

32. Miyazaki T, Kato H, Nakajima M, Sohda M, Fukai Y, Masuda N, Manda R, Fukuchi M, Tsukada K and Kuwano H: FAK overexpression is correlated with tumour invasiveness and lymph node metastasis in oesophageal squamous cell carcinoma. Br J Cancer 89: 140-145, 2003.
33. Golubovskaya VM: Targeting FAK in human cancer: From finding to first clinical trials. Front Biosci (Landmark Ed) 19: 687-706, 2014.

34. Ocak S, Chen H, Callison C, Gonzalez AL and Massion PP Expression of focal adhesion kinase in small-cell lung carcinoma. Cancer 118: 1293-1301, 2012.

35. Ji HF, Pang D, Fu SB, Jin Y, Yao L, Qi JP and Bai J: Overexpression of focal adhesion kinase correlates with increased lymph node metastasis and poor prognosis in non-small-cell lung cancer. J Cancer Res Clin Oncol 139: 429-435, 2013.

(i) (2) This work is licensed under a Creative Commons C) AY NG Attribution-NonCommercial-NoDerivatives 4.0 International (CC BY-NC-ND 4.0) License. 